Revista Historia de la Educación Colombiana. No. 11. (ISSN: 0123-7756) (pp. 9-31). 2008.

APA citation style: Benavides B., Jorge E. (2008). Una Aproximación Interdisciplinar del Análisis Crítico del Discurso (ACD) al Estudio de la Historia. Revista Historia de la Educación Colombiana (11), 9-31.

\title{
Una Aproximación Interdisciplinar del Análisis Crítico del Discurso (ACD) al Estudio de la Historia
}

\author{
Jorge E. Benavides B. (joelbebu@gmail.com) \\ Departamento de Lingüística e Idiomas \\ Universidad de Nariño, San Juan de Pasto, Colombia
}

\section{RESUMEN}

El presente artículo tiene como objetivo introducir la consideración del nuevo campo del análisis crítico del discurso (ACD) como forma complementaria al estudio de la historia. Intenta abordar algunos conceptos interdisciplinares para la incorporación de este enfoque investigativo de reciente desarrollo, con orígenes en la teoría crítica, la lingüística crítica y el análisis del discurso al campo de la historia. Se presenta su característica central en la consideración del discurso como acontecimiento histórico, como expresión sociocultural condicionado por las estructuras sociales y de creencia de grupos de poder que dominan sobre otros haciendo del discurso, determinante del contexto social y a la vez transmisor de ideologías. Se presenta las prácticas sociales paralelamente a las prácticas discursivas existiendo una relación dialéctica entre ambas. Las relaciones de poder, dominio, hegemonía y especialmente la ideología son las áreas de trabajo en las que se concentra el ACD y cuyo campo de acción implica el recurso interdisciplinario y transdisciplinario en áreas como la psicología, la sociología, la economía, la política y la

\footnotetext{
${ }^{\mathrm{i}}$ Este artículo constituye una parte del componente teórico del trabajo de tesis doctoral "Análisis Crítico del Discurso Oficial de las Nuevas Tecnologías de la Información y la Comunicación (NTIC) para la Universidad Colombiana en el Contexto de la Globalización". A través de los documentos oficiales del gobierno de finales del siglo XX se presenta la estrecha relación entre la historia y el campo de los estudios del discurso, el lenguaje y la lingüística como ciencias interdisciplinares para el acercamiento al estudio de la ideología y el poder a través del análisis crítico del discurso (ACD).
} 
Revista Historia de la Educación Colombiana. No. 11. (ISSN: 0123-7756) (pp. 9-31). 2008.

educación (entre otras) relacionándose con la historia para poder describir, interpretar y explicar a través de los discursos (escritos y orales) las relaciones históricas entre el lenguaje y la sociedad.

\section{PALABRAS CLAVE}

Lenguaje, análisis crítico del discurso ( $A C D)$, discurso, historia, poder, ideología.

\section{ABSTRACT}

The objective of this article is to invite the reader to consider the new field of critical discourse analysis (CDA) as a complementary way to the study of history. It intends to approach several interdisciplinary concepts for the incorporation of critical discourse analysis, a relatively recent theoretical and practical approach of discourse analysis based on critical theory, critical linguistics and discourse analysis into history. It centers its focus on the consideration of discourse as an event, a socio-cultural expression conditioned by social structures and belief systems of groups of power acting upon others and conditioning the social context while being the transmitter of ideology. It presents social practices in parallel to discursive ones thereby creating a dialectal relationship between them. The relations of power, hegemony, dominance, and especially, ideology, are the main concerns of critical discourse analysis thereby making room for interdisciplinary and transdisciplinary joint efforts to research: psychology, sociology, politics, economics, and education, among a few others are related to history in order to describe, interpret and explain the historical relationships between society and language.

\section{KEYWORDS:}

Language, critical discourse analysis (CDA), discourse, history, power, ideology.

\section{Introducción}

Este relativamente nuevo campo investigativo del análisis crítico del discurso tiene como base a la lingüística y particularmente al campo del lenguaje y el discurso como disciplinas fundantes en la transición desde la 
Revista Historia de la Educación Colombiana. No. 11. (ISSN: 0123-7756) (pp. 9-31). 2008.

lingüística descriptiva a la generativa y al posterior desarrollo de la pragmática y a la sociolingüística. Esta relación del campo del lenguaje con el de la sociología se ha visto fortalecido con el trabajo de Halliday (1978) y su lingüística funcional sistémica donde se enfatiza el proceso de la transmisión del mensaje en forma completa e histórica. De esta manera, la lingüística llega a relacionarse estrechamente con el campo del estudio crítico del discurso atravesando el desarrollo de la lingüística crítica (Kress y Hodge, 1979).

Los inicios del análisis crítico del discurso ( $A C D$, de aquí en adelante) se empezaron a entrever con la publicación de varias obras que ligaba a la lingüística con aspectos de la sociología, y la ideología como el caso de Lenguaje y Control (Fowler et al., 1979), Lenguaje como Ideología (Kress y Hodge, 1979) y Lenguaje y Poder (Fairclough, 1989), influenciados por nuevos enfoques tanto lingüísticos, filosóficos, como sociológicos como los de Wittgenstein (1953), Pêcheux (1975), y Foucault (1972). Su influencia pasó al campo de la sociolingüística, la pragmática, la comunicación y la antropología lingüística que han sido importantes en la concreción del ACD (Gumperz y Hymes, 1972; Labov, 1972; Sinclair y Coulthard, 1975) enriqueciendo así el campo del discurso como producto socio-cultural en sus diferentes manifestaciones.

Se podría decir que el ACD se deriva de varias tradiciones que han enfatizado el 'giro lingüístico' en las ciencias sociales como los estudios sobre el discurso de Benveniste (1971), Foucault (1972), el análisis del discurso de Gumperz y Hymes (1972), la lingüística crítica de Fowler et al. (1979); y la lingüística funcional de Halliday y Hasan (1989). El 'giro lingüístico' como desarrollo de la filosofía occidental del lenguaje y la importancia de éste en las ciencias sociales fue el reconocimiento dado a la capacidad del lenguaje para construir y constituir la realidad. Uno de los supuestos más importantes del ACD es el potencial de su utilización para determinar cómo el lenguaje sirve de herramienta socio-cultural con la que se crean, se transmiten, y se mantienen relaciones de poder, dominio, hegemonía, privilegio, etc., a través de la ideología en las interacciones de grupos de poder como en el caso de lo que sucede con la comunicación en las instituciones sociales (Foucault, 1972; Bourdieu, 1984). 
Revista Historia de la Educación Colombiana. No. 11. (ISSN: 0123-7756) (pp. 9-31). 2008.

\section{El lenguaje y el discurso y su relación con la investigación en historia}

\subsection{El estudio del lenguaje en la historia}

El interés de la historia por la lingüística para estudios de tipo interdisciplinario comienza prácticamente con los esfuerzos de Marc Bloch y Lucien Febvre por enriquecer los análisis y complementar el método histórico. En La societé Feodale (Bloch, 1939), por ejemplo, se percibe el aporte fundamental de la lingüística en los análisis de los términos y las nociones que de éstos se derivan. Los términos y nociones analizadas en esta obra se presentan como sistemas de representaciones que funcionan 'ideológicamente' en una sociedad determinada espacio-temporalmente y se convierten en un objeto de estudio, a saber: el léxico y las nociones utilizadas desde una perspectiva histórica. Lucien Febvre también destacó la importancia del conocimiento lingüístico y filológico para el estudio de la historia en el nivel del análisis interdisciplinario. Su estudio sobre Civilisation: le mot et l'idée (Febvre, 1930) resalta también la importancia del conocimiento semántico en el estudio y análisis de las palabras, expresiones y conceptos para el campo de la historia de las ideas. Además en la publicación de Combats pour l'histoire Febvre (1953) establece la necesidad de la interdisciplinariedad de estos dos campos sosteniendo que la lengua es la 'vía cardinal' de acceso al aspecto social para la historia y de la misma manera pide la cooperación de los lingüistas para que los historiadores puedan servirse de ese conocimiento. Denomina 'Alianzas' y 'apoyos' de la historia a la cooperación entre la lingüística y la historia cuando sostiene que

...hay necesidad de la colaboración de aquellos 'semantistas' quienes, restituyéndonos la historia de las palabras particularmente cargadas de sentido escriben del mismo puño capítulos precisos de la historia de las ideas. $^{1}$

Importante también es considerar la aparición de la 'Nueva historia' desde comienzos de los sesenta, especialmente como un nuevo dominio 'La historia de las mentalidades' que se concentraría en un nuevo objeto de estudio como son los sentimientos, las sensibilidades, las expresiones, las creencias, las actitudes, los imaginarios ${ }^{2}$, los signos y los símbolos. En este sentido ya no sería el factor económico el más importante en este nuevo dominio sino el factor mental que implicaría la colaboración y el apoyo de otras disciplinas como la antropología, la psicología, la sociología y 
Revista Historia de la Educación Colombiana. No. 11. (ISSN: 0123-7756) (pp. 9-31). 2008.

especialmente la lingüística. Tampoco es ya tan importante la consideración de las fechas y las épocas sino el estudio de las personas como grupo, dentro de determinado contexto. Grammaire des civilizations de Fernand Braudel (1987 [1963]) establece el nuevo objeto de estudio como la representación del mundo y de las cosas de cada época particular: su mentalidad colectiva que anima y pinta la masa entera de la sociedad, enfatizando de esta manera las creencias e ideologías de un grupo de personas en un momento histórico dado. Esto le llevaría a sostener que,

Mucho más que los accidentes o las circunstancias históricas y sociales de una época, ella [la mentalidad] es el fruto de herencias lejanas, de creencias, de miedos, de inquietudes antiguas a menudo casi inconscientes... ${ }^{3}$

También el mismo Braudel, refiriéndose directamente al nuevo campo del estudio de las mentalidades en el concepto de la Nueva Historia dirá que "Esta mentalidad que dicta las actitudes, orienta las opciones, sostiene los prejuicios, inclina los movimientos de una sociedad es eminentemente un hecho de civilización." ${ }^{4}$ Inclusive, a nivel del estudio de las representaciones de una sociedad como el que hacía Georges Duby (1961) en Histoire des mentalités se hace alusión a la importancia del lenguaje llamando las 'herramientas' a los aspectos del lenguaje utilizados para entender las expresiones que los individuos obtienen de grupos sociales. Destaca de la misma manera la importancia del lenguaje en la historia; lenguaje sin el cual no se podría indagar en la misma conciencia del hombre:

\footnotetext{
Entre estas 'herramientas' sobre las que el estudio en efecto se impone, vienen en primer lugar el lenguaje como los diversos medios de expresión que el individuo recibe del grupo social donde vive y que sirven de marco a toda su vida mental. ¿Cómo penetrar en la conciencia de los hombres de tal medio, cómo explicar su conducta, las relaciones que cultivan, tratar de ver el mundo y al otro a través de sus ojos, sin conocer el vocabulario, que emplean o más aun los vocabularios, ya que muchos hombres utilizan varios, adaptados a diferentes grupos...? ${ }^{5}$
}

De esta manera, se puede notar que la relación e integración investigativa de la lingüística con la historia comienza en el nivel de la lexicografía aplicada al análisis de las mentalidades y de las ideas. Dupront (1964) explícitamente valora los campos de la semántica y la semiótica dentro de la ciencia del lenguaje para el estudio de la psiquis colectiva de un grupo 
Revista Historia de la Educación Colombiana. No. 11. (ISSN: 0123-7756) (pp. 9-31). 2008.

determinado, por los que su conocimiento por parte del historiador es necesario y se podría decir, también, natural. Otros estudios en los que el aporte de la lingüística al estudio de la historia ha jugado un papel importante han sido realizados durante la segunda mitad del siglo XX, como los de Lebrun (1971); y Vovelle (1973) en los que se aprecia un acercamiento al estudio de la ideología como fenómeno social a través del recurso en la lingüística, (lexicografía) especialmente referido al estudio del lenguaje en los documentos históricos.

Los estudios de tipo histórico en el nivel del discurso propiamente dicho llegarían inicialmente con la publicación de los trabajos de Michel Foucault Historia de la locura (1961) y El nacimiento de la clínica (1963). Aunque estos no comprendían el análisis del discurso como objeto de estudio en sí sino más bien de los contextos y de las condiciones en las que éste se materializaba si constituyó un aporte diferente a la concepción del discurso tal como había sido previamente considerado por la lingüística descriptiva o el estructuralismo del círculo de Praga, con Ferdinand de Saussure, cuando se veía como un conjunto de oraciones a nivel oral y escrito que describían algo. El discurso sale prácticamente de las fronteras de la lingüística para insertarse en forma más directa con el aspecto social en su correspondencia histórica. Con la publicación de El orden del discurso (Foucault, 1971) se destaca la restitución al discurso de su carácter de 'acontecimiento', en el cual se pone en duda la preponderancia por la verdad total en cuanto a la soberanía del significante.

A finales de la década de los años setenta publicaciones como La nueva historia de Jacques Le Goff (1978) incluía esta nueva área interdisciplinaria de la historia conjuntamente con el estudio del discurso donde se hace un llamado a considerar esta implementación en el estudio histórico. Por otro lado, la lingüística crítica se desarrolló a finales de los años setenta como una disciplina en la que el rigor de los análisis sobre pasaban lo que hasta ese momento se había hecho. Los investigadores de la lingüística crítica, se centraron en la investigación del uso del lenguaje en 'entornos institucionales' con la introducción de un enfoque que integraba una perspectiva histórica (el enfoque histórico del discurso), lo cual produjo investigaciones sobre las practicas discursivas en contextos institucionales que eventualmente indicarían el camino integral del análisis crítico del discurso (Wodak, 2003b). 
Revista Historia de la Educación Colombiana. No. 11. (ISSN: 0123-7756) (pp. 9-31). 2008.

\subsection{El concepto de la explicación-comprensión}

Al concepto de la explicación-comprensión, se lo ha denominado recientemente como la nueva fase de la operación historiográfica después de la fase documental, donde esta ya aparece estrechamente integrada y donde debido al documento que se increpa o pregunta es necesario dar una explicación (Ricoeur, 2000). Sin embargo, atendiendo no necesariamente a la relación pregunta-respuesta de tipo causal, sino al de comprensión del hecho se hace propio la modelación de los conceptos que ya son conocidos dentro del ámbito de la historia (descripción, comprensión, interpretación y explicación) a los del análisis del discurso con orientación crítica y que parten desde la lingüística y la hermenéutica para acercarse a los mismos sobre la base de la teoría del discurso. Según Gadamer (1977) la interpretación es la forma en la que se realiza la comprensión, es decir, no habría comprensión si no existe una interpretación del texto como discurso (hechos o practicas discursivas). A su vez, la explicación, como una forma de dar razones sobre algo (un hecho, después de su descripción) permitiría una mejor comprensión. De ahí que explicar implicaría comprender mejor, o sea que la explicación implica una determinada interpretación y comprensión de un texto o documento, a través de su discurso como acontecimiento. Se podría asegurar entonces que la explicación de una interpretación progresivamente permite una mayor comprensión de un evento discursivo. De manera similar, Calvo y Avila (1991) sostienen que la explicación es un desarrollo analítico de la comprensión donde se aprecia que la primera también implica un proceso de análisis, es decir, no se puede explicar algo sino se ha realizado un análisis previo. Así pues el análisis se presenta a través de la explicación y ésta a su vez es ya una interpretación que conlleva la comprensión de un fenómeno (hecho, discurso, acontecimiento).

En el evento de conocimiento de tipo histórico, por ejemplo, se podría recurrir a la noción que ofrece Aróstegui (2001) sobre la explicación cuando sostiene que "el resultado de todo proceso de conocimiento sujeto a un método es siempre, desde luego, una explicación. Una explicación que en su grado de mayor perfección es una teoría" (p. 338). Tomado de esta manera, lo que pretende el ACD como producto final es la teorización a través del discurso, del estado de cosas en un contexto dado. En este caso particular se estaría preguntando sobre la 
Revista Historia de la Educación Colombiana. No. 11. (ISSN: 0123-7756) (pp. 9-31). 2008.

forma como funciona el poder a través de la ideología desde el discurso. En otras palabras, el ACD pretende determinar la configuración del poder y la ideología en el discurso como acontecimiento social de un determinado contexto.

\section{El lenguaje, el discurso y la filosofía}

El impulso dado a la lingüística por filósofos del lenguaje como Ludwig Wittgenstein, Roman Jakobson, Zellig Harris, John Austin, y John Searle, entre otros, sobre la importancia del lenguaje como acción discursiva y social en el nivel de la frase, la oración, la proposición y el enunciado converge con el interés en el nivel del discurso como objeto de estudio donde éste hace parte de un contexto social al que afecta pero que a su vez es afectado por él en el orden del discurso. Por otro lado, Karl Marx y Max Weber, conocidos representantes de la escuela moderna de la filosofía y la sociología occidentales vieron en el determinismo económico una de las causas de opresión social donde la economía se ve como una fuerza determinante en muchos aspectos de la existencia del hombre. Otros filósofos más contemporáneos como Theodor Adorno, Herbert Marcuse y Max Horkheimer dieron inicio con sus trabajos a lo que se conoce como "teoría crítica" (Escuela de Frankfurt) tomando aportes de diferentes disciplinas de apoyo, una de las cuales fue precisamente la lingüística. Esta área intrigaba a los filósofos e investigadores sociales en el sentido de poder determinar si la lengua se encontraba también haciendo parte, o inclusive como base, económica de la superestructura cultural; si estaba determinada por las condiciones materiales o si en su lugar, ésta las determinaba.

Es especialmente importante el giro que desde la filosofía se dio a la concepción de la expresión de lo social a través del lenguaje. El mejor ejemplo de esto se puede encontrar desde la antigüedad mediante el trabajo de Platón en los diálogos socráticos como el Fedro y el Menón, por ejemplo donde a Sócrates le interesaba saber lo que la gente pensaba sobre los conceptos 'amor' y 'virtud' respectivamente. Sin embargo, como en el caso de la 'virtud' no le interesaba que la gente le diera ejemplos de 'virtud' sino lo que era en realidad la 'virtud' como cualidad del concepto presente en todas las posibles realizaciones del mismo, es decir la definición abstracta del concepto. Lo que le interesaba era la posibilidad de la definición exacta de las cosas como cuando se definía lo que era un 
Revista Historia de la Educación Colombiana. No. 11. (ISSN: 0123-7756) (pp. 9-31). 2008.

cuadrado en términos matemáticos. De esta manera Sócrates afirmaba que no se podía dar una definición precisa de las cosas que preguntaba. El problema se empezó a ver entonces en el lenguaje mismo.

En la época moderna este problema también lo tocó Wittgenstein (1961 [1921]), especialmente en su primer trabajo, el Tractatus logicophilosophicus en el sentido que muchas cosas no se pueden definir a través del lenguaje en la forma como se hace con los conceptos matemáticos y lógicos. La teoría del significado como imagen de la realidad a través del lenguaje fue precisamente la posibilidad de tener en el lenguaje una imagen precisa y lógica de la realidad en ciertas proposiciones mas no en otras al no poderlas expresar en forma precisa y sobre lo cual era mejor callar pues no presentaban como otras una relación directa con lo expresado por el lenguaje. De alguna manera solo cierto tipo de proposiciones darían una imagen de la realidad y la lógica de algún hecho. Así, para Wittgenstein en el Tractatus había dos tipos de proposiciones evidentes, en, las deductivas y las inductivas. Las primeras podían ser falsas o verdaderas, es decir tautologías, como en una ecuación matemática (una igualdad). Las proposiciones inductivas por otro lado se encontraban en el lado más contingente del espectro discursivo. Estas no representaban aspectos del mundo: 'yo soy asi', 'te lo prometo', 'vendré mañana para verla' se entendían solo mirando al mundo en una determinada situación.

Es su trabajo póstumo, Investigaciones filosóficas, Wittgenstein (1953) se apartaría de su teoría lógico-matemático de las proposiciones como imágenes del mundo y giraría hacia una concepción más contingente de las expresiones que ocurrían en el mundo. Propuso entonces el lenguaje como en un 'juego' ('juegos del lenguaje'), un conjunto de prácticas sociales que se entrecruzan pero que a la vez no tienen una única línea de significación y que solo se permite comprenderlas en forma abstracta. De esta manera, el lenguaje como un 'juego' de juegos no tendría una característica general que cubriera a todas las posibles expresiones en su definición. Según la nueva concepción, el lenguaje está subsumido en el concepto de 'juegos del lenguaje' y éstos no son idénticos en sus reglas, situaciones o aplicaciones, lo cual produce diferentes tipos de ambigüedad y 'sin-sentidos' al querer aplicar reglas de un juego a otro, o inclusive imponerlas. A pesar de que sí existe una cierta consistencia en los juegos como sistemas, no había una sola característica lingüística que aplicara a todas las expresiones o a todos 
Revista Historia de la Educación Colombiana. No. 11. (ISSN: 0123-7756) (pp. 9-31). 2008.

los juegos. Cada juego se realizaría en momentos, contextos y tiempos determinados y diferentes. No existiría ningún tipo de juego que sea idéntico, sino solo en algunas pequeñas coincidencias entre ellos. Los significados de las palabras (conceptos, significado y sentido) tendrían que aprenderse desde el uso mismo del término y en el contexto en el que suceden (en el juego). De esta manera era necesario preguntar no sobre el significado de una palabra sino sobre su uso (cómo la utiliza la gente -lo que hacemos con ella) y esto estaría imbricado en la contingencia de las acciones humanas.

\section{El desarrollo del análisis crítico del discurso}

\subsection{La lingüística crítica (LC) en el desarrollo del análisis crítico del discurso}

Este enfoque lingüístico crítico dentro de la historia del $A C D$ tiene una importancia social innegable ya que hace parte del trabajo seminal que condujo al desarrollo de la lingüística funcional sistémica de Halliday (1973, 1975, 1978), los trabajos de Fowler et al. (1979), la influencia parcial del círculo de Praga, del sociólogo educacional Basil Bernstein (cfr. Bernstein, 1973), del semiótico Roland Barthes y especialmente la influencia de los lingüistas Edward Sapir y Benjamin Lee-Whorf. La lingüística critica que nació del trabajo y preocupación de un grupo de investigadores y lingüistas en los años setenta basa su enfoque en la concepción integral y funcional del lenguaje y se diferencia de la sociolingüística y de la corriente chomskyana de la época en la concepción de la diferencia entre forma y contenido; estructura y uso, forma y función como base para el análisis del discurso. La lingüística crítica insistió en el análisis del lenguaje como una unidad de textos y expresiones y dentro de contextos sociales reales para ofrecer una explicación del 'cómo' y el 'por qué' de las diferencias de uso, con referencia al poder y la ideología.

El texto se consideró entonces no solo como afectado políticamente sino como la realización de una función ideológica en estrecha relación con los contextos en los que eran históricamente producidos. Sin embargo se descuidó el estudio de la relación que podría existir entre los cambios en el discurso y su relación con los cambios de tipo social y cultural como fondo de los primeros. En este sentido, y en su desarrollo, la lingüística crítica 
Revista Historia de la Educación Colombiana. No. 11. (ISSN: 0123-7756) (pp. 9-31). 2008.

integró el pensamiento de Michel Foucault sobre el discurso, las instituciones y el poder, y la preocupación por el feminismo como fenómeno social. La lingüística crítica desarrolló también una dimensión intertextual y subjetiva en los procesos discursivos sobre el cambio para lograr una posición flexible de análisis lingüístico que pudiera integrarse a la teoría social.

\section{2. El $A C D$ en el ámbito de la sociología}

El ACD intenta dar una explicación sociológica del uso del lenguaje puesto que su interés está puesto en la ideología, las relaciones sociales y las relaciones entre el texto y el contexto. Además, investiga las categorías del discurso como una forma de poder y como instrumento de la construcción social de la realidad. Además proporciona un énfasis de tipo sociopolítico, lo que no implica que la complejidad de la metodología se pase por alto sino que por el contrario, muestra los avances lingüísticos puestos al análisis de los problemas de tipo social y político, cultural e histórico.

La teoría critica se convirtió entonces en un conjunto de teorías cuya función era la de localizar y confrontar aspectos de poder, dominio, hegemonía, y privilegio, y las formas o medios en que la economía, la política, la religión, la educación, etc. contribuían, en la reproducción, transformación, o mantenimiento de los sistemas sociales y de grupos de poder. Un supuesto importante del que parte la teoría crítica es que el 'pensamiento' está mediado por relaciones de poder históricamente constituidas. Sostiene además que ningún hecho es neutral (o naturalmente puro en el sentido de que algo sucede históricamente sin ninguna clase de intervención o mediación cultural de algún tipo) sino que éstos son consecuencia de direccionamientos ideológicos que operan desde el 'poder' en primera instancia y esa ideología está en su base determinada, transmitida y mantenida a través del lenguaje como discurso a nivel oral y escrito. Los discursos están rodeados de contextos en los que siempre han existido grupos de poder y de dominio sobre otros. Este poder y dominio conlleva a un acceso diferenciado de unos grupos sobre otros en términos de pensamiento, de información, de conocimiento, y de bienes y servicios, por ejemplo.

A pesar de que el giro lingüístico compromete a la sociolingüística y la pragmática, los contextos sociales y políticos no se teorizan ni analizan 
Revista Historia de la Educación Colombiana. No. 11. (ISSN: 0123-7756) (pp. 9-31). 2008.

adecuadamente en ese ámbito, y así dan por sentados factores como el nivel socio-económico y la influencia de las instituciones sociales sobre los individuos (Fowler et al., 1979). Contrariamente, el ACD se nutre de la tradición de la teoría crítica con su enfoque en las diferencias entre los grupos y clases sociales. Teun van Dijk (1993), por ejemplo, manifiesta que en el ACD la comprensión de las aspectos sociales y sus relaciones e influencias externas a través del análisis del discurso son fundamentales, por ejemplo en el fenómeno de las discriminación racial (racismo), el dominio del mercado (consumismo, mercantilismo) y Fairclough (1989) la orientación empresarial de la educación (comodificación, mercantilización). En el campo de la educación se puede incluir las influencias externas que afectan o determinan a los grupos o instituciones del estado (la escuela, la universidad, etc.) y que se manifiestan en los discursos producidos por estos. En este sentido el ACD se constituye en una forma de investigación reflexiva.

\subsection{Las características del análisis crítico del Discurso (ACD)}

Una aproximación al análisis crítico del discurso tiene que incluir tanto el componente lingüístico como su contraparte social, o sea, tanto la forma como la función. Esto tiene básicamente que ver, como se había manifestado anteriormente, con el análisis sobre el poder, el dominio, la hegemonía y la ideología que las instituciones ejercen (crean, reproducen, transmiten y mantienen) mediante los discursos producidos en un contexto social o político determinado; discursos institucionales, políticos, y mediáticos, entre otros. Wodak (2003a) manifiesta que la crítica no se ocupa de aspectos puramente lingüísticos sino de aspectos como el poder y la ideología es decir, del lenguaje como 'práctica social' en el contexto de su uso donde

... el lenguaje es también un medio de dominación y una fuerza social. Sirve para legitimar las relaciones del poder organizado. En la medida en que las legitimaciones de las relaciones de poder, [...] no estén articuladas, [...] el lenguaje es también ideológico ${ }^{6}$.

De la misma manera Foucault (1972) consideraba que el texto como discurso es, en su expresión más amplia, la unidad básica de comunicación y el objeto de estudio en el ambiente social e histórico. 
Revista Historia de la Educación Colombiana. No. 11. (ISSN: 0123-7756) (pp. 9-31). 2008.

Tanto para Ruth Wodak, Norman Fairclough como para Teun van Dijk, tres de los pioneros del $A C D$, el espíritu y la dimensión critica que 'encarna la academia', es decir, la universidad como institución social en su función y razón de ser es la que le imprime la motivación intrínseca de este nuevo tipo de estudios. Esto se confirma en el sentido de que el ACD y la lingüística crítica (LC) comparten los campos del análisis del discurso, de la semiótica y de la lingüística (van Dijk, 1993; Fairclough y Wodak, 1997). Estas relaciones de causa y efecto, siendo tan difíciles de notar en la actividad humana (y que usualmente se ocultan o distorsionan) se harían visibles, como lo expresa Fairclough (1995b) a través del análisis crítico del discurso, mediante el cual se revelarían el poder y la ideología. En otras palabras, los usos del lenguaje a través del discurso revelarían las relaciones de poder e inequidad en el tratamiento de aspectos sociales, culturales, educativos en donde una ideología estaría dominando y la de otro grupo muy posiblemente resistiendo. El lenguaje como medio de dominación también ha sido planteado como aspecto a investigar por pensadores como Habermas (1982) quien elabora sobre el lenguaje como un medio ideológico de dominio; Fairclough (1995b) sobre los contenidos ideológicos en el lenguaje; van Dijk (1998) sobre el carácter multidisciplinario de la ideología y el lenguaje; y Larraín (1979), y Boudon (1989) sobre el carácter amplio e incisivo de la ideología en la comunicación. Habermas (1992), Habermas y Blazek, (1987) reiteran la afectación del poder en el discurso a diferencia del concepto chomskiano de una 'situación ideal del hablante' cuando oponen a ésta la 'situación real del discurso' en donde las situaciones discursivas pueden distorsionarse por las estructuras de poder. Estas y su contraparte, la resistencia, marcan por un lado, las formas discursivas naturales como convenciones establecidas que todos aceptan, conformándose lo que Foucault consideraba como 'orden del discurso' y por otro las formas de ruptura de esas convenciones, las cuales Fowler et al. (1979) llamarían 'actos creativos'.

El poder y la ideología juegan un papel central en el ACD puesto que se considera a los discursos 'ideológicamente afectados' a diferencia de la 'ingenua' consideración de la arbitrariedad de sus signos. Estos conceptos van de la mano con las formas lingüísticas en el campo de la retórica, la lingüística textual, y la sociolingüística. En este orden de ideas últimamente los medios de comunicación se han venido intensificando como objetos de estudio en cuanto a los discursos que producen, transmiten, mantienen y/o 
Revista Historia de la Educación Colombiana. No. 11. (ISSN: 0123-7756) (pp. 9-31). 2008.

modifican. Es ahí donde el indiscutible poder de los medios masivos de comunicación ha inspirado gran cantidad de estudios críticos como el caso de los análisis de los mensajes e imágenes estereotipados, racistas y sexistas tanto a nivel de texto como a nivel semiótico visual (Fowler et al., 1979).

\subsection{El enfoque metodológico del ACD}

Desarrollar un enfoque analítico apropiado desde los multiformes significados en cuestión es una tarea difícil y compleja dado que existen en primer lugar una buena cantidad de definiciones de discurso que se intersectan y que se formulan desde variados puntos de vista teóricos en las diferentes disciplinas. Sin embargo, una definición sencilla de discurso desde una mirada social, es la concepción de que el lenguaje es una parte intrínseca de la sociedad y no algo externo a ella, además que el lenguaje estaría condicionado por otros factores no lingüísticos de la sociedad, el poder y la ideología (Fairclough, 1989). Dentro de los límites de esta definición simplificada existen otras explicaciones sobre el discurso que se han desarrollado, alrededor de una gran cantidad de disciplinas académicas, especialmente desde las ciencias sociales donde especial atención se ha puesto a los efectos del lenguaje como reflexión de una perspectiva construccionista.

Según Fairclough (1992a, 1995a) no se puede desconocer la importancia del lenguaje en las operaciones de poder y de hegemonía como foco de interés de la comunidad académica mundial. Bajo las condiciones contemporáneas de la modernidad alta hacia la postmodernidad es importante un foco crítico en la consideración de la producción y reproducción del conocimiento como discurso (y viceversa), y dando particular énfasis a las formas en las que la ideología está investida en el lenguaje (van Dijk, 1998). La relación entre ideología y las representaciones del conocimiento son de preocupación central y pueden afrontarse desde una perspectiva del análisis del discurso. Como categoría analítica, el discurso puede contribuir a determinar la construcción de identidad social (sujeto), la construcción de relaciones sociales entre la gente y las instituciones; y la construcción de sistemas de conocimiento y creencia (Fairclough, 1992a).

Una teoría del discurso puede contribuir también a comprender cómo las identidades sociales son modeladas y alteradas en el tiempo por la gente y 
Revista Historia de la Educación Colombiana. No. 11. (ISSN: 0123-7756) (pp. 9-31). 2008.

especialmente por los grupos hegemónicos y cómo éstos difieren de los discursos de la resistencia. El análisis del discurso visto como un enfoque tanto teórico como metodológico representa un programa promisorio de investigación en ciencias sociales y humanas cuyo propósito principal es examinar la aparente estabilidad de los patrones de relaciones sociales, y sus influencias desde los diversos ámbitos del quehacer humano. En este enfoque se atribuye considerable importancia a los actores sociales de las políticas de cambio, a los grupos que lo producen, a los que lo interpretan y lo explican a través del discurso. El ACD intenta capturar las conexiones entre el lenguaje y la sociedad: las prácticas sociales y las prácticas discursivas en sus contextos históricos determinados.

\subsection{Los niveles del análisis crítico del discurso}

Para van Dijk (2000) en el ACD se presentan dos tipos de análisis, uno a nivel 'micro' y otro a nivel 'macro'. El primero tiene que ver con las estructuras lingüísticas y sus relaciones en el texto, sin embargo, el nivel macro se asocia a los niveles sociales y culturales y el establecimiento, reproducción y legitimación del poder. Para Fairclough (1989, 1992b) existen tres dimensiones: la dimensión 'micro' o análisis textual (lingüística textual) que tiene que ver con las formas y el significado de éstas en el texto, la dimensión 'meso' intermedia o practica discursiva que se enfoca en la producción e interpretación del texto, y la dimensión 'macro', o sea, la práctica sociocultural que opera a un estrato más amplio de análisis social.

En el enfoque tridimensional de Fairclough, el más utilizado a nivel general, la primera dimensión trata al discurso como texto y su análisis se remite a los rasgos y organización de las estructuras del discurso. Ejemplo de esto son la selección del léxico (vocabulario, metáfora), la gramática (transitividad, pasividad, modalidad), cohesión (marcadores, conjunciones), y la estructura textual (representación de papeles o roles, estilo de narración). Este es el análisis de tipo lingüístico formal y se aplica a todos los géneros desde el periodístico o reportaje de noticias (donde el uso, por ejemplo, de la voz pasiva puede intencionadamente ocultar el agente de un hecho), hasta los discursos formales u oficiales. La segunda dimensión de este enfoque tridimensional tiene que ver con la práctica discursiva, es decir, el discurso como algo que se produce, se transmite y se consume en la sociedad. Esto implica el análisis de patrones de vocabulario, gramática, cohesión, con especial énfasis en la coherencia y la intertextualidad lo que 
Revista Historia de la Educación Colombiana. No. 11. (ISSN: 0123-7756) (pp. 9-31). 2008.

determina en última instancia el contexto del discurso. En este campo se encuentran aspectos discursivos como los tipos de discurso, el 'estilo', 'género', 'registro', y la forma de representación discursiva. La tercera dimensión es la consideración del discurso como práctica social que tiene que ver con los efectos y patrones ideológicos, de poder y los procesos hegemónicos del discurso: la lucha por la hegemonía y el poder representada en los discursos puede entreverse en el uso y función del discurso, teniendo siempre en cuenta las otras dos dimensiones (explicación-interpretación).

Con esto se determinaría que a medida que las hegemonías cambian también los hacen sus discursos representativos. La forma en que se representa el discurso es indicio de un nuevo establecimiento u orden de cosas representado en el 'orden del discurso' (Fairclough, 1989, 1995b). Esta tercera dimensión 'macro' es la parte en la que el ACD se centra para la identificación de las relaciones entre el lenguaje y la sociedad, y el análisis de las prácticas del discurso para hacer del mismo visiblemente como algo socialmente condicionado y que a la vez condiciona lo social. Es una característica importante que los cambios económicos, sociales, políticos y culturales de la modernidad, que existen primeramente como cambios discursivos y también como procesos, se sucedan en gran medida, discursivamente.

De esta manera, el enfoque tridimensional del análisis crítico del discurso propuesto por Fairclough implica moverse entre la descripción, la interpretación y la explicación de los patrones que a nivel institucional presentan los discursos en el campo social. Estas tres dimensiones, similarmente con el tratamiento dado por van Dijk están en una disposición jerárquica tal que el análisis 'macro' abarca lo 'micro' pero que como proceso empieza por lo 'micro' lo cual indica la complejidad de análisis en el nivel más amplio del discurso donde el contexto y la interdiscursividad juegan un papel importante.

\subsection{El carácter cualitativo del ACD}

La preparación cualitativa del ACD dentro del estudio del contexto implica un análisis de tipo histórico para determinar cómo las formas del lenguaje llegan a organizarse o realizar determinados vínculos sociales. Todos los discursos son históricos y solo pueden entenderse por referencia a su 
Revista Historia de la Educación Colombiana. No. 11. (ISSN: 0123-7756) (pp. 9-31). 2008.

contexto (Parker, 2004). Esto es importante puesto que el análisis del lenguaje de un texto (discurso) no se debe tratar como si éste hubiera aparecido de la nada: todo lo que tiene significado (sentido para las personas) deber tener algunas precondiciones 'históricas' para que haya sido escrito o producido como una imagen del mundo, sea en mayor o en menor grado ideológica. En este sentido se promueve el giro de lo científico natural y cuantitativo hacia lo crítico social como posibilidad cualitativa de análisis y como una verdadera alternativa al enfoque positivista de la historia. Desde la posición subjetiva se hace necesario un desafío en cada individuo a las 'supuestas' verdades del 'sentido común' bajo esquemas tan persuasivos como el capitalismo, la globalización, el neoliberalismo, el mercantilismo, entre otros, de nuestra época actual.

Además del contexto, la intertextualidad y la recontextualización, el ACD utiliza como factores extralingüísticos: el poder y la ideología en la cultura, ya que se incluirían aspectos sociales, políticos, y económicos haciéndose necesario un procedimiento interdisciplinario y eventualmente transdisciplinario de los análisis. El recurso a la intertextualidad es decisivo en el ACD puesto que se hace necesario el análisis de las relaciones con otros textos, y otros contextos de influencia, además de las características lingüísticas de su análisis. El contexto en el que los textos, (documentos o fuentes, por ejemplo) se configuran y realizan comprende principalmente en forma histórica las relaciones con otros textos y otros contextos para producir el 'enfoque histórico del discurso' que según Meyer (2003) es deudor de la teoría critica, y donde "... encuentra su punto focal en el campo de la política, ámbito en el que intenta desarrollar marcos conceptuales para el discurso político" ${ }^{7}$

Meyer sostiene que el procedimiento metodológico que sigue el ACD es de tipo hermenéutico, a pesar de que no hay unanimidad en todos los investigadores del discurso. A diferencia de las ciencias naturales en las que el centro son las explicaciones causales, en el ACD la hermenéutica (descripción, interpretación y explicación) puede comprenderse como un enfoque metodológico para determinar las relaciones significativas de poder en el texto y entre los textos. Sin embargo, la interpretación hermenéutica requiere de una detallada documentación con la que prima lo intensivo sobre lo extensivo de los análisis. En la misma dirección de van Dijk (1995), Meyer (2003), también indica que el ACD no debe entenderse 
Revista Historia de la Educación Colombiana. No. 11. (ISSN: 0123-7756) (pp. 9-31). 2008.

como un método sino más bien como un enfoque, es decir, "como algo que adquiere consistencia en varios planos y que cada uno de esos planos exige realizar un cierto número de selecciones." 8

\section{Características del enfoque histórico del discurso}

En resumen se podría decir que los tres conceptos que se enfatizan en un estudio de ACD con interés histórico son: el de poder, el de ideología y el de historia. Teniendo en cuenta que el discurso se estructura según factores de 'dominio' ejercido por lo socialmente establecido, es necesario considerar el planteamiento de Wodak (2003a) sobre el aspecto social e histórico del mismo:

... todo discurso es un objeto históricamente producido e interpretado, esto es, que se halla situado en el tiempo y en el espacio, y de que las estructuras de dominancia están legitimadas por las ideologías de grupos [de poder], el complejo enfoque que proponentes de la LC [Lingüística Crítica] y el ACD permite analizar las presiones provenientes de arriba y las posibilidades de resistencia a las desiguales relaciones de poder que aparecen en forma de convenciones sociales ${ }^{9}$ (énfasis mío).

Para el enfoque histórico del discurso dentro del marco del $A C D$, Wodak establece diez puntos como referencia para este tipo de análisis:

1. El enfoque es interdisciplinar, en varios planos: en la teoría, en los equipos de investigación y en la práctica.

2. El enfoque se orienta hacia los problemas sociales; no se centra solo en elementos específicamente lingüísticos.

3. Tanto la teoría como la metodología son eclécticas, esto es, se incorporan las teorías y los métodos que resultan útiles para comprender y explicar el objeto sometido a investigación.

4. Como condición previa para cualquier análisis y teorización ulterior, el estudio incluye siempre el trabajo de campo y la etnografía con el fin de explorar el objeto de investigación.

5. El enfoque es abductivo o emergente: es necesario realizar un constante movimiento de ida y vuelta entre la teoría y los datos empíricos.

6. Se estudian múltiples variedades discursivas y múltiples espacios públicos como contexto, y se investigan las relaciones intertextuales e interdiscursivas. La recontextualización es el proceso más importante para vincular esas variedades discursivas, junto con los temas y los argumentos.

7. El contexto histórico se analiza siempre y se incorpora a la interpretación de los discursos y los textos. 
Revista Historia de la Educación Colombiana. No. 11. (ISSN: 0123-7756) (pp. 9-31). 2008.

8. Las categorías y las herramientas para el análisis se definen en función de todos estos pasos y procedimientos, en consonancia con el problema que se está investigando.

9. Las grandes teorías actúan como fundamento [...]. Para el análisis específico, las teorías de rango medio contribuyen mejor a los objetivos analíticos.

10. El objetivo es la práctica. Los resultados deberían quedar a disposición de los expertos de los diferentes campos, y, como segundo paso, aplicarse con el fin de eventualmente cambiar ciertas prácticas discursivas y sociales. ${ }^{10}$

\section{Conclusión}

El ACD ofrece una particular oportunidad de contribución en el estudio interdisciplinario y transdisciplinario de los eventos históricos mediados por el discurso en relación con el estudio de las relaciones de poder e ideología a nivel socio-cultural. El hombre está expuesto a diario a todo tipo de imágenes, mensajes, signos, símbolos y texto como discursos en su labor cotidiana en una sociedad que impone una determinada orientación ideológica a través de sus mensajes, ya sea a nivel de los medios masivos como en el ambiente académico e investigativo. Esta exposición dentro de un concepto de discurso 'semiótico' como la organización del lenguaje en el que se construyen vínculos sociales hace que reflexionemos sobre la 'real' intensión de muy buena cantidad de mensajes y discursos que median entre los diferentes eventos y acontecimientos sociales. Ante la cadena del discurso, entre las palabras e imágenes (las nuestras y las de otros) en las que creemos, nos tendríamos que preguntar entonces de qué manera el discurso se produce, ser transforma y se mantiene ideológica e históricamente haciendo que las relaciones de poder producidas incluyan a ciertos grupos sociales y excluyan a otros, es decir, que el discurso no solo describe el mundo y lo representa sino que también lo constituye y lo construye contextual e ideológicamente. 


\section{NOTAS}

${ }^{1}$ FEBVRE, Lucien (1953). Combats pour I'histoire. Paris: Librairie Armand Colin. p. 219.

2 Los 'Imaginarios colectivos' como los desarrollados en forma más extensa en el trabajo de Paul Ricoeur. (1989). Ideología y utopía. Barcelona: Gedisa.

3 BRAUDEL, Fernand (1987). Grammaire des civilisations. Paris: ArthaudFlammarion. p. 54.

${ }^{4}$ Ibidem.

5 DUBY, Georges (1961). Histoire des mentalités. En Samaran (Ed.), L'Histoire et ses méthodes (pp. 911-966). Paris: Gallimard. p. 953.

${ }^{6}$ WODAK, Ruth (2003a). De qué trata el análisis crítico del discurso (ACD)? En Wodak y Meyer (Eds.), Métodos de análisis crítico del discurso (pp. 17-34). Barcelona: Gedisa. Citando a Habermas. (1982). Conocimiento e interés. Madrid: Taurus. p. 19.

7 MEYER, Michael (2003). Entre la teoría, el método y la política; la ubicación de los enfoques relacionados con el ACD. En Wodak y Meyer (Eds.), Métodos de análisis crítico del discurso (pp. 35-59). Barcelona: Gedisa. p. 46.

8 Ibid. p. 35.

${ }^{9}$ WODAK. pp. 19-20.

10 WODAK. (2003b). El enfoque histórico del discurso. En Wodak y Meyer (Eds.), Métodos de análisis crítico del discurso (pp. 101-142). Barcelona: Gedisa. pp. 109-110.

\section{REFERENCIAS}

ARÓSTEGUI, Julio. (2001). La investigación histórica: teoría y método. Barcelona: Crítica (Grijalbo Mondadori).

BENVENISTE, Emile. (1971). Problems in general linguistics. Coral Gables, Florida: University of Miami Press.

BERNSTEIN, Basil. (1973). Class, codes and control. London: Routledge \& Kegan Paul.

BLOCH, Marc. (1939). La Societé Feodale. La Formation des Liens de Dépendance. Paris: Éditions Albin Michel. 
Revista Historia de la Educación Colombiana. No. 11. (ISSN: 0123-7756) (pp. 9-31). 2008.

BOUDON, Raymond. (1989). The analysis of ideology. Chicago: University of Chicago Press.

BOURDIEU, Pierre. (1984). Homo academicus. Paris: Ed. de Minuit.

BRAUDEL, Fernand. (1987). Grammaire des civilisations. Paris: ArthaudFlammarion.

CALVo MARTíneZ, Tomás y Avila Crespo, Remedios (Eds.). (1991). Paul Ricoeur: Los Caminos de la Interpretación (1 ed.). Barcelona: Editorial Anthropos.

DUBY, Georges. (1961). "Histoire des mentalités". En C. Samaran (Ed.), L'Histoire et ses méthodes (pp. 911-966). Paris: Gallimard.

DUPRONT, Alphonse. (1964). L'histoire et l'historien. Paris: Librairie Arthème Fayard.

FAIRCLOUGH, Norman. (1989). Language and power. London; New York: Longman.

FAIRCLOUGH, Norman. (1992a). Discourse and social change. Cambridge, UK: Polity Press.

FAIRCLOUGH, Norman. (1992b). Discourse and Text: Linguistic and Intertextual Analysis within Discourse Analysis. Discourse and Society, 3(2), 193-217.

FAIRCLOUGH, Norman. (1995a). "Critical Discourse Analysis and the Marketization of Public Discourse: The Universities". En N. Fairclough (Ed.), Critical discourse analysis: the critical study of language (pp. 130-166). London; New York: Longman.

FAIRCLOUGH, Norman. (1995b). Critical discourse analysis: the critical study of language. London; New York: Longman.

FAIRCLOUGH, Norman, y Wodak, Ruth. (1997). "Análisis Crítico del Discurso". En T. A. van Dijk (Ed.), El Discurso como Interacción Social (Vol. 2, pp. 367-404). Barcelona: Gedisa.

FEBVRE, Lucien. (1930). Civilisation: le mot et l'idée. Paris: Centre International de Synthèse.

FEBVRE, Lucien. (1953). Combats pour l'histoire. Paris: Librairie Armand Colin.

FOUCAULT, Michel. (1971). L'ordre du discours. Paris: Gallimard.

FOUCAULT, Michel. (1972). The archaeology of knowledge (1a. ed.). New York: Pantheon Books.

FOUCAULT, Michel. (1967) [1961]. Historia de la locura en la época clásica. México, D.F.: Fondo de Cultura Económica. 
Revista Historia de la Educación Colombiana. No. 11. (ISSN: 0123-7756) (pp. 9-31). 2008.

FOUCAULT, Michel. (1966) [1963]. El nacimiento de la clínica. Madrid: Siglo XXI.

FOUCAULT, Michel. (1987) [1971]. El orden del discurso. Barcelona: Trsquets

FOWLER, Roger, Hodge, Bob, Kress, Gunther, y Trew, Tony. (1979). Language and control. London; Boston: Routledge \& K. Paul.

GADAMER, Hans-Georg. (1977). Verdad y método: fundamentos de una hermenéutica filosófica. Salamanca: Sígueme.

GUMPERZ, John Joseph, y Hymes, Dell H. (1972). Directions in sociolinguistics: the ethnography of communication. New York: Holt.

HABERMAS, Jürgen. (1982). Conocimiento e interés. Madrid: Taurus.

HABERMAS, Jürgen. (1992). Ciencia y técnica como "ideología" (2a. ed.). Madrid: Tecnos.

HABERMAS, Jurgen, y Blazek, John R. (1987). The idea of the University: Learning Processes. New German Critique(41), 3-22.

HALLIDAY, M. A. K., y Hasan, Ruqaiya. (1989). Language, context, and text : aspects of language in a social-semiotic perspective (2a. ed.). Oxford ; New York: Oxford University Press.

HALLIDAY, Michael. (1973). Explorations in the functions of language. London: Edward Arnold.

HALLIDAY, Michael. (1975). Learning how to mean, explorations in the development of language. [London]: Arnold.

HALLIDAY, Michael. (1978). Language as social semiotic: the social interpretation of language and meaning. London: Edward Arnold.

KRESS, Gunther, y Hodge, Bob. (1979). Language as ideology. London; Boston: Routledge \& Kegan Paul.

LARRAÍN, Jorge. (1979). The concept of ideology. Athens: University of Georgia Press.

LAVOB, William (1972). Language in the inner city: studies in the black english vernacular. Philadelphia: University of Pennsylvania Press.

LE GOFF, Jacques. (1978). La nouvelle histoire. Paris: Complexe.

LEBRUN, Francois. (1971). Les hommes et la mort en Anjou aux 17e et 18 e siècles essai de démographie et de psychologie historiques. Paris: Mouton.

MEYER, Michael (2003). "Entre la teoría, el método y la política; la ubicación de los enfoques relacionados con el ACD" (Trad. A. Fernández Tomás y B. Eguibar). En R. Wodak y M. Meyer (Eds.), Métodos de análisis crítico del discurso (pp. 35-59). Barcelona: Gedisa. 
Revista Historia de la Educación Colombiana. No. 11. (ISSN: 0123-7756) (pp. 9-31). 2008.

PARKER, Ian (2004). "This world demands our attention: lan Parker in conversation with Dimitris Papadopoulos". Forum: Qualitative Social Research, 5(3), 1-10.

PÊCHEUX, Michel. (1975). Analyse du discours langue et idéologies. Paris: Didier-Larousse.

RICOEUR, Paul. (1989). Ideología y utopía (Trad. A. Bixio 1a ed.). Barcelona: Gedisa.

RICOEUR, Paul. (2000). La Mémoire, I'histoire, l'oubli. Paris: Éditions du Seuil.

SINCLAIR, John y COULTHARD, Malcolm (1975). Towards an analysis of discourse: the english used by teachers and pupils. London: Oxford University Press.

VAN DIJK, Teun. (1993). "Principles of Critical Discourse Analysis". Discourse and Society, 4(2), 249-283.

VAN DIJK, Teun. (1995). "Discourse Semantics and Ideology". Discourse Society, 6(2), 243-289.

VAN DIJK, Teun. (1998). Ideología: un enfoque multidisciplinario (Trad. L. Berrone de Blanco). Barcelona: Editorial Gedisa, S.A.

VAN DIJK, Teun. (Ed.). (2000). El Discurso como Interacción Social. Estudios sobre el discurso: introducción multidisciplinaria. (Vol. 2). Barcelona: Editorial Gedisa S.A.

VOVELLE, Michel. (1973). Piété baroque et déchristianisation en Provence au XVIIle siècle: les attitudes devant la mort d'après les clauses des testaments. Paris: Plon.

WITTGENSTEIN, Ludwig. (1953). Philosophical investigations. Oxford: B. Blackwell.

WITTGENSTEIN, Ludwig. (1961) [1921]. Tractatus logico-philosophicus. London: Routledge \& Kean Paul.

WODAK, Ruth. (2003a). "De qué trata el análisis crítico del discurso (ACD)?” En R. Wodak y M. Meyer (Eds.), Métodos de análisis crítico del discurso (pp. 17-34). Barcelona: Gedisa.

WODAK, Ruth. (2003b). "El enfoque histórico del discurso". En R. Wodak y M. Meyer (Eds.), Métodos de análisis crítico del discurso (pp. 101142). Barcelona: Gedisa. 